\title{
Blow-up analysis for parabolic $p$-Laplacian equations with a gradient source term
}

Juntang Ding ${ }^{1 *}$ (D)

\section{"Correspondence:}

djuntang@sxu.edu.cn

'School of Mathematical Sciences, Shanxi University, Taiyuan 030006, P.R. China

\section{Springer}

\begin{abstract}
In this work, we deal with the blow-up solutions of the following parabolic $p$-Laplacian equations with a gradient source term:

$$
\begin{cases}(b(u))_{t}=\nabla \cdot\left(|\nabla u|^{p-2} \nabla u\right)+f\left(x, u,|\nabla u|^{2}, t\right) & \text { in } \Omega \times\left(0, t^{*}\right), \\ \frac{\partial u}{\partial n}=0 & \text { on } \partial \Omega \times\left(0, t^{*}\right), \\ u(x, 0)=u_{0}(x) \geq 0 & \text { in } \bar{\Omega},\end{cases}
$$
\end{abstract}

where $p>2$, the spatial domain $\Omega \subset \mathbb{R}^{N}(N \geq 2)$ is bounded, and the boundary $\partial \Omega$ is smooth. Our research relies on the creation of some suitable auxiliary functions and the use of the differential inequality techniques and parabolic maximum principles. We give sufficient conditions to ensure that the solution blows up at a finite time $t^{*}$. The upper bounds of the blow-up time $t^{*}$ and the upper estimates of the blow-up rate are also obtained.

MSC: $35 \mathrm{~K} 65 ; 35 \mathrm{~B} 40$

Keywords: Blow-up solution; Parabolic $p$-Laplacian equation; Gradient source term

\section{Introduction}

The blow-up solutions of parabolic $p$-Laplician equations have been studied by many authors (see, for instance, [1-10]). In this work, we research the blow-up solutions of the following parabolic $p$-Laplacian equations with a gradient source term:

$$
\begin{cases}(b(u))_{t}=\nabla \cdot\left(|\nabla u|^{p-2} \nabla u\right)+f\left(x, u,|\nabla u|^{2}, t\right) & \text { in } \Omega \times\left(0, t^{*}\right), \\ \frac{\partial u}{\partial n}=0 & \text { on } \partial \Omega \times\left(0, t^{*}\right), \\ u(x, 0)=u_{0}(x) \geq 0 & \text { in } \bar{\Omega} .\end{cases}
$$

In (1.1), $p>2$, the spatial domain $\Omega \subset \mathbb{R}^{N}(N \geq 2)$ is bounded, the boundary $\partial \Omega$ is smooth, and $t^{*}$ is blow-up time, $b(s)$ is a $C^{3}\left(\overline{\mathbb{R}_{+}}\right)$function with $b^{\prime}(s)>0, s \in \overline{\mathbb{R}_{+}}, f(x, s, r, t)$ is a nonnegative $C^{1}\left(\Omega \times \overline{\mathbb{R}_{+}} \times \overline{\mathbb{R}_{+}} \times \mathbb{R}_{+}\right)$function, and $u_{0}(x)$ is a nonnegative $C^{2}(\bar{\Omega})$ function satisfying $\frac{\partial u_{0}(x)}{\partial n}=0, x \in \partial \Omega$.

(c) The Author(s) 2020. This article is licensed under a Creative Commons Attribution 4.0 International License, which permits use, sharing, adaptation, distribution and reproduction in any medium or format, as long as you give appropriate credit to the original author(s) and the source, provide a link to the Creative Commons licence, and indicate if changes were made. The images or other third party material in this article are included in the article's Creative Commons licence, unless indicated otherwise in a credit line to the material. If material is not included in the article's Creative Commons licence and your intended use is not permitted by statutory regulation or exceeds the permitted use, you will need to obtain permission directly from the copyright holder. To view a copy of this licence, visit http://creativecommons.org/licenses/by/4.0/. 
As far as I know, there are many papers on the blow-up problems of parabolic equations with a gradient term (see, for instance, [11-23]). For the sake of research (1.1), we mainly focus on the papers $[12,22]$. In [22], Zhang et al. researched the following problems:

$$
\begin{cases}(b(u))_{t}=\nabla \cdot\left(\rho\left(|\nabla u|^{p}\right)|\nabla u|^{p-2} \nabla u\right)+h(x) k(t) f(u) & \text { in } \Omega \times\left(0, t^{*}\right), \\ u(x, t)=0 & \text { on } \partial \Omega \times\left(0, t^{*}\right), \\ u(x, 0)=u_{0}(x) \geq 0 & \text { in } \bar{\Omega} .\end{cases}
$$

In (1.2), $p \geq 2$, the spatial domain $\Omega \subset \mathbb{R}^{N}(N \geq 2)$ is bounded, and the boundary $\partial \Omega$ is smooth. They derived some conditions which ensure the solution of (1.2) blows up in a finite $t^{*}$ or exists globally. Moreover, an upper bound and a lower bound of the blow-up time were also specified when the blow-up occurs. Their research relied on using differential inequality techniques. In [12], Ding et al. studied the following problems:

$$
\begin{cases}(b(u))_{t}=\Delta u+f\left(x, u,|\nabla u|^{2}, t\right) & \text { in } \Omega \times\left(0, t^{*}\right) \\ \frac{\partial u}{\partial n}=r(u) & \text { on } \partial \Omega \times\left(0, t^{*}\right) \\ u(x, 0)=u_{0}(x)>0 & \text { in } \bar{\Omega} .\end{cases}
$$

In (1.3), the spatial domain $\Omega \subset \mathbb{R}^{N}(N \geq 2)$ is bounded, and the boundary $\partial \Omega$ is smooth. By combining the parabolic maximum principles and the differential inequality techniques, they obtained sufficient conditions for the existence of the blow-up solution and the global solution of (1.3). In addition, the upper bounds of the blow-up time $t^{*}$ and the upper estimates of the blow-up rate were also given.

Inspired by the above two research works, in this paper we study the blow-up solutions of (1.1). Since the source term of the equation in (1.1) contains a gradient term, and the source term of the equation in (1.2) does not, the research methods in [22] cannot be used to research (1.1). In this paper, we use the method in [12] to study (1.1). In other words, we combine the parabolic maximum principles with the differential inequality techniques to study (1.1). The difficulty in using this research method is that some suitable auxiliary functions need to be constructed. We note that although the source terms in equations of (1.1) and (1.3) are the same, the boundary conditions are different. Therefore, the auxiliary functions in the paper [12] are not suitable for researching (1.1). In this paper, we need to construct some auxiliary functions that are completely different from those in [12] to study the blow-up solution of (1.1). We give sufficient conditions to guarantee that the solution of (1.1) blows up at a finite time $t^{*}$. The upper bounds of the blow-up time $t^{*}$ and the upper estimates of the blow-up rate are also obtained.

For convenience, in this paper we use a comma to represent partial derivative and adopt the summation convention, for example,

$$
u, i, u_{j} u, i j=\sum_{i=1}^{N} \sum_{j=1}^{N} \frac{\partial u}{\partial x_{i}} \frac{\partial u}{\partial x_{j}} \frac{\partial^{2} u}{\partial x_{i} \partial x_{j}}
$$




\section{The main result and its proof}

Due to the need to study the blow-up solution of (1.1), we define the following two constants:

$$
\begin{aligned}
& \alpha=\min _{x \in \bar{\Omega}} \frac{\nabla \cdot\left(\left|\nabla u_{0}\right|^{p-2} \nabla u_{0}\right)+f\left(x, u_{0},\left|\nabla u_{0}\right|^{2}, 0\right)}{\mathrm{e}^{u_{0}}}, \\
& \beta=\inf _{(x, s, t) \in \Omega \times \mathbb{\mathbb { R }}_{+} \times \mathbb{R}_{+}} \frac{f(x, s, 0, t)}{\mathrm{e}^{s}},
\end{aligned}
$$

where $u_{0}$ is the initial value of (1.1). For the same purpose, we also construct two auxiliary functions as follows:

$$
\begin{aligned}
& G(x, t)=b^{\prime}(u) u_{t}-\alpha \mathrm{e}^{u}, \quad(x, t) \in \bar{\Omega} \times\left[0, t^{*}\right), \\
& H(s)=\int_{s}^{+\infty} \frac{b^{\prime}(\tau)}{\mathrm{e}^{\tau}} \mathrm{d} \tau, \quad s \in \overline{\mathbb{R}_{+}},
\end{aligned}
$$

where $u(x, t)$ is a nonnegative $C^{3}\left(\Omega \times\left(0, t^{*}\right)\right) \cap C^{2}\left(\bar{\Omega} \times\left[0, t^{*}\right)\right)$ solution of (1.1). Now we have

$$
H^{\prime}(s)=-\frac{b^{\prime}(s)}{\mathrm{e}^{s}}<0, \quad s \in \mathbb{R}_{+},
$$

which implies that the function $H$ has an inverse function $H^{-1}$. The following Theorem 2.1 is the main result of the blow-up solution to (1.1).

Theorem 2.1 Let $u$ be a nonnegative $C^{3}\left(\Omega \times\left(0, t^{*}\right)\right) \cap C^{2}\left(\bar{\Omega} \times\left[0, t^{*}\right)\right)$ solution of (1.1). Assume the following four assumptions are true:

(i)

$$
\beta \geq \alpha>0
$$

(ii)

$$
\int_{M_{0}}^{+\infty} \frac{b^{\prime}(\tau)}{\mathrm{e}^{\tau}} \mathrm{d} \tau<+\infty, \quad M_{0}=\max _{x \in \bar{\Omega}} u_{0}(x)
$$

(iii) For $s \in \overline{\mathbb{R}_{+}}$,

$$
(p-1)\left(\frac{1}{b^{\prime}(s)}\right)^{\prime}+(p-2) \frac{1}{b^{\prime}(s)} \geq 0, \quad\left(\frac{1}{b^{\prime}(s)}\right)^{\prime \prime}+2\left(\frac{1}{b^{\prime}(s)}\right)^{\prime}+\frac{1}{b^{\prime}(s)} \geq 0 .
$$

(iv) $\operatorname{For}(x, s, r, t) \in \Omega \times \overline{\mathbb{R}_{+}} \times \overline{\mathbb{R}_{+}} \times \mathbb{R}_{+}$,

$$
\begin{aligned}
& f_{r}(x, s, r, t) \geq 0, \quad f_{t}(x, s, r, t) \geq 0, \\
& \frac{f_{s}(x, s, r, t)}{b^{\prime}(s)}-(p-1) f(x, s, r, t)\left[\left(\frac{1}{b^{\prime}(s)}\right)^{\prime}+\frac{1}{b^{\prime}(s)}\right] \geq 0 .
\end{aligned}
$$


Then, $u(x, t)$ must blow up at a finite time $t^{*}$ and

$$
t^{*} \leq \frac{1}{\alpha} \int_{M_{0}}^{+\infty} \frac{b^{\prime}(\tau)}{\mathrm{e}^{\tau}} \mathrm{d} \tau,
$$

as well as

$$
u(x, t) \leq H^{-1}\left(\alpha\left(t^{*}-t\right)\right), \quad(x, t) \in \bar{\Omega} \times\left[0, t^{*}\right) .
$$

Proof By directly calculating the auxiliary function $G(x, t)$ defined in (2.3), we have

$$
G,,_{i}=b^{\prime \prime} u_{t} u,_{i}+b^{\prime} u_{t, i}-\alpha \mathrm{e}^{u} u,,_{i}
$$

and

$$
G_{, i j}=b^{\prime \prime \prime} u_{t} u,,_{i} u, b_{j}+b^{\prime \prime} u_{t, j} u,,_{i}+b^{\prime \prime} u_{t, i} u,,_{j}+b^{\prime \prime} u_{t} u,_{i j}+b^{\prime} u_{t, i j}-\alpha \mathrm{e}^{u} u,_{i} u,,_{j}-\alpha \mathrm{e}^{u} u,_{i j} .
$$

By (2.10), we get

$$
\Delta G=G_{i i}=b^{\prime \prime \prime}|\nabla u|^{2} u_{t}+2 b^{\prime \prime}\left(\nabla u \cdot \nabla u_{t}\right)+b^{\prime \prime} u_{t} \Delta u+b^{\prime} \Delta u_{t}-\alpha \mathrm{e}^{u}|\nabla u|^{2}-\alpha \mathrm{e}^{u} \Delta u
$$

Making use of the first equation of (1.1), we derive

$$
\begin{aligned}
G_{t}= & \left(b^{\prime}(u) u_{t}\right)_{t}-\alpha \mathrm{e}^{u} u_{t}=\left[(b(u))_{t}\right]_{t}-\alpha \mathrm{e}^{u} u_{t} \\
= & {\left[\nabla \cdot\left(|\nabla u|^{p-2} \nabla u\right)+f(x, u, q, t)\right]_{t}-\alpha \mathrm{e}^{u} u_{t} } \\
= & {\left[|\nabla u|^{p-2} \Delta u+(p-2)|\nabla u|^{p-4} u,_{i} u,,_{j} u, i j+f(x, u, q, t)\right]_{t}-\alpha \mathrm{e}^{u} u_{t} } \\
= & (p-2)|\nabla u|^{p-4}\left(\nabla u \cdot \nabla u_{t}\right) \Delta u+|\nabla u|^{p-2} \Delta u_{t} \\
& +(p-2)(p-4)|\nabla u|^{p-6}\left(\nabla u \cdot \nabla u_{t}\right) u,{ }_{i} u,{ }_{j} u, i j \\
& +2(p-2)|\nabla u|^{p-4} u_{t, i} u,{ }_{j} u, i j \\
& +(p-2)|\nabla u|^{p-4} u, u_{i} u_{, j} u_{t, i j}+f_{u} u_{t}+2 f_{q}\left(\nabla u \cdot \nabla u_{t}\right)+f_{t}-\alpha \mathrm{e}^{u} u_{t},
\end{aligned}
$$

where $q=|\nabla u|^{2}$. It follows from (2.10)-(2.12) that

$$
\begin{aligned}
& \frac{|\nabla u|^{p-2}}{b^{\prime}} \Delta G+(p-2) \frac{|\nabla u|^{p-4}}{b^{\prime}} u,{ }_{i} u,{ }_{j} G,{ }_{i j}-G_{t} \\
& =(p-1) \frac{b^{\prime \prime \prime}}{b^{\prime}}|\nabla u|^{p} u_{t}+2(p-1) \frac{b^{\prime \prime}}{b^{\prime}}|\nabla u|^{p-2}\left(\nabla u \cdot \nabla u_{t}\right)+\frac{b^{\prime \prime}}{b^{\prime}}|\nabla u|^{p-2} u_{t} \Delta u \\
& -\alpha(p-1) \frac{\mathrm{e}^{u}}{b^{\prime}}|\nabla u|^{p}-\alpha \frac{\mathrm{e}^{u}}{b^{\prime}}|\nabla u|^{p-2} \Delta u+(p-2) \frac{b^{\prime \prime}}{b^{\prime}}|\nabla u|^{p-4} u_{t} u,_{i} u,{ }_{j} u, i j \\
& -\alpha(p-2) \frac{\mathrm{e}^{u}}{b^{\prime}}|\nabla u|^{p-4} u,_{i} u,{ }_{j} u,_{i j}-(p-2)|\nabla u|^{p-4}\left(\nabla u \cdot \nabla u_{t}\right) \Delta u \\
& -(p-2)(p-4)|\nabla u|^{p-6}\left(\nabla u \cdot \nabla u_{t}\right) u,{ }_{i} u,{ }_{j} u,{ }_{i j}-2(p-2)|\nabla u|^{p-4} u_{t,{ }_{i}} u,{ }_{j} u,{ }_{i j} \\
& +\left(\alpha \mathrm{e}^{u}-f_{u}\right) u_{t}-2 f_{q}\left(\nabla u \cdot \nabla u_{t}\right)-f_{t} .
\end{aligned}
$$

With (2.9), we obtain

$$
u_{t},{ }_{i}=\frac{1}{b^{\prime}} G,{ }_{i}-\frac{b^{\prime \prime}}{b^{\prime}} u_{t} u,,_{i}+\alpha \frac{\mathrm{e}^{u}}{b^{\prime}} u,_{i}
$$


and

$$
\nabla u_{t}=\frac{1}{b^{\prime}} \nabla G-\frac{b^{\prime \prime}}{b^{\prime}} u_{t} \nabla u+\alpha \frac{\mathrm{e}^{u}}{b^{\prime}} \nabla u
$$

We insert (2.14) and (2.15) into (2.13) to derive

$$
\begin{aligned}
& \frac{|\nabla u|^{p-2}}{b^{\prime}} \Delta G+(p-2) \frac{|\nabla u|^{p-4}}{b^{\prime}} u,{ }_{i} u,{ }_{j} G, i j \\
& +\frac{1}{b^{\prime}}\left((p-2)(p-4)|\nabla u|^{p-6} u,_{i} u,,_{j} u,_{i j}+(p-2)|\nabla u|^{p-4} \Delta u-2(p-1) \frac{b^{\prime \prime}}{b^{\prime}}|\nabla u|^{p-2}\right. \\
& \left.+2 f_{q}\right)(\nabla u \cdot \nabla G)+2(p-2) \frac{|\nabla u|^{p-4}}{b^{\prime}} u,{ }_{i} u,{ }_{i j} G,{ }_{i}-G_{t} \\
& =\left((p-1) \frac{b^{\prime \prime \prime}}{b^{\prime}}-2(p-1) \frac{\left(b^{\prime \prime}\right)^{2}}{\left(b^{\prime}\right)^{2}}\right)|\nabla u|^{p} u_{t}+\left(2 \alpha(p-1) \frac{b^{\prime \prime} \mathrm{e}^{u}}{\left(b^{\prime}\right)^{2}}-\alpha(p-1) \frac{\mathrm{e}^{u}}{b^{\prime}}\right)|\nabla u|^{p} \\
& +(p-1) \frac{b^{\prime \prime}}{b^{\prime}}|\nabla u|^{p-2} u_{t} \Delta u-\alpha(p-1) \frac{\mathrm{e}^{u}}{b^{\prime}}|\nabla u|^{p-2} \Delta u \\
& +(p-1)(p-2) \frac{b^{\prime \prime}}{b^{\prime}}|\nabla u|^{p-4} u_{t} u,{ }_{i} u,{ }_{j} u, i j-\alpha(p-1)(p-2) \frac{\mathrm{e}^{u}}{b^{\prime}}|\nabla u|^{p-4} u,_{i} u,{ }_{j} u,_{i j} \\
& +\left(\alpha \mathrm{e}^{u}-f_{u}\right) u_{t}+2 \frac{f_{q} b^{\prime \prime}}{b^{\prime}}|\nabla u|^{2} u_{t}-2 \alpha \frac{f_{q} \mathrm{e}^{u}}{b^{\prime}}|\nabla u|^{2}-f_{t} .
\end{aligned}
$$

By the first equation of (1.1), we have

$$
|\nabla u|^{p-2} \Delta u=b^{\prime} u_{t}-(p-2)|\nabla u|^{p-4} u,{ }_{i} u,{ }_{j} u,{ }_{i j}-f .
$$

We insert (2.17) into (2.16) to get

$$
\begin{aligned}
& \frac{|\nabla u|^{p-2}}{b^{\prime}} \Delta G+(p-2) \frac{|\nabla u|^{p-4}}{b^{\prime}} u,,_{i} u,,_{j} G_{i j} \\
& \quad+\frac{1}{b^{\prime}}\left((p-2)(p-4)|\nabla u|^{p-6} u,,_{i} u,{ }_{j} u,{ }_{i j}+(p-2)|\nabla u|^{p-4} \Delta u-2(p-1) \frac{b^{\prime \prime}}{b^{\prime}}|\nabla u|^{p-2}\right. \\
& \left.\quad+2 f_{q}\right)(\nabla u \cdot \nabla G)+2(p-2) \frac{|\nabla u|^{p-4}}{b^{\prime}} u,_{i} u,_{i j} G,_{i}-G_{t} \\
& =\left((p-1) \frac{b^{\prime \prime \prime}}{b^{\prime}}-2(p-1) \frac{\left(b^{\prime \prime}\right)^{2}}{\left(b^{\prime}\right)^{2}}\right)|\nabla u|^{p} u_{t}+\left(2 \alpha(p-1) \frac{b^{\prime \prime} \mathrm{e}^{u}}{\left(b^{\prime}\right)^{2}}-\alpha(p-1) \frac{\mathrm{e}^{u}}{b^{\prime}}\right)|\nabla u|^{p} \\
& \quad+(p-1) b^{\prime \prime}\left(u_{t}\right)^{2}-\left(\alpha(p-2) \mathrm{e}^{u}+f_{u}+(p-1) \frac{f b^{\prime \prime}}{b^{\prime}}\right) u_{t} \\
& \quad+\alpha(p-1) \frac{f \mathrm{e}^{u}}{b^{\prime}}+2 \frac{f_{d} b^{\prime \prime}}{b^{\prime}}|\nabla u|^{2} u_{t}-2 \alpha \frac{f_{d} \mathrm{e}^{u}}{b^{\prime}}|\nabla u|^{2}-f_{t} .
\end{aligned}
$$

It follows from (2.3) that

$$
u_{t}=\frac{1}{b^{\prime}} G+\alpha \frac{\mathrm{e}^{u}}{b^{\prime}}
$$


We insert (2.19) into (2.18) to obtain

$$
\begin{aligned}
\frac{|\nabla u|^{p-2}}{b^{\prime}} \Delta G+(p-2) \frac{|\nabla u|^{p-4}}{b^{\prime}} u,_{i} u,_{j} G,_{i j} \\
+\frac{1}{b^{\prime}}\left((p-2)(p-4)|\nabla u|^{p-6} u,_{i} u,{ }_{j} u,_{i j}+(p-2)|\nabla u|^{p-4} \Delta u-2(p-1) \frac{b^{\prime \prime}}{b^{\prime}}|\nabla u|^{p-2}\right. \\
\left.+2 f_{q}\right)(\nabla u \cdot \nabla G)+2(p-2) \frac{|\nabla u|^{p-4}}{b^{\prime}} u,{ }_{i} u,,_{i j} G,_{i} \\
+\left\{\frac{1}{\left(b^{\prime}\right)^{2}}\left[\left(2(p-1) \frac{\left(b^{\prime \prime}\right)^{2}}{b^{\prime}}-(p-1) b^{\prime \prime \prime}\right)|\nabla u|^{p}-2 f_{q} b^{\prime \prime}|\nabla u|^{2}\right]\right. \\
\left.+\frac{1}{b^{\prime}}\left[\alpha \mathrm{e}^{u}\left(p-2-2(p-1) \frac{b^{\prime \prime}}{b^{\prime}}\right)+f_{u}+(p-1)^{\prime} \frac{f b^{\prime \prime}}{b^{\prime}}-(p-1) \frac{b^{\prime \prime}}{b^{\prime}} G\right]\right\} G-G_{t} \\
=-\alpha(p-1) \mathrm{e}^{u}\left[\left(\frac{1}{b^{\prime}}\right)^{\prime \prime}+2\left(\frac{1}{b^{\prime}}\right)^{\prime}+\frac{1}{b^{\prime}}\right]|\nabla u|^{p}-\alpha^{2} \mathrm{e}^{2 u}\left[(p-1)\left(\frac{1}{b^{\prime}}\right)^{\prime}+(p-2) \frac{1}{b^{\prime}}\right] \\
\quad-\alpha \mathrm{e}^{u}\left\{\frac{f_{u}}{b^{\prime}}-(p-1) f\left[\left(\frac{1}{b^{\prime}}\right)^{\prime}+\frac{1}{b^{\prime}}\right]\right\}-2 \alpha \mathrm{e}^{u} f_{q}\left[\left(\frac{1}{b^{\prime}}\right)^{\prime}+\frac{1}{b^{\prime}}\right]|\nabla u|^{2}-f_{t} .
\end{aligned}
$$

From assumptions (2.7) and (2.8) we know that the right-hand side of equality (2.20) is nonpositive. So now we have

$$
\begin{aligned}
& \frac{|\nabla u|^{p-2}}{b^{\prime}} \Delta G+(p-2) \frac{|\nabla u|^{p-4}}{b^{\prime}} u,_{i} u,,_{j},_{, i j} \\
& \quad+\frac{1}{b^{\prime}}\left((p-2)(p-4)|\nabla u|^{p-6} u,_{i} u,,_{j} u,_{i j}+(p-2)|\nabla u|^{p-4} \Delta u-2(p-1) \frac{b^{\prime \prime}}{b^{\prime}}|\nabla u|^{p-2}\right. \\
& \left.\quad+2 f_{q}\right)(\nabla u \cdot \nabla G)+2(p-2) \frac{|\nabla u|^{p-4}}{b^{\prime}} u,_{i} u,,_{i j} G,_{i} \\
& +\left\{\frac{1}{\left(b^{\prime}\right)^{2}}\left[\left(2(p-1) \frac{\left(b^{\prime \prime}\right)^{2}}{b^{\prime}}-(p-1) b^{\prime \prime \prime}\right)|\nabla u|^{p}-2 f_{q} b^{\prime \prime}|\nabla u|^{2}\right]\right. \\
& \left.+\frac{1}{b^{\prime}}\left[\alpha \mathrm{e}^{u}\left(p-2-2(p-1) \frac{b^{\prime \prime}}{b^{\prime}}\right)+f_{u}+(p-1) \frac{f b^{\prime \prime}}{b^{\prime}}-(p-1) \frac{b^{\prime \prime}}{b^{\prime}} G\right]\right\} G \\
& \quad-G_{t} \leq 0 \quad \text { in } \Omega \times\left(0, t^{*}\right) .
\end{aligned}
$$

Combining (2.21) and parabolic maximum principles ([24], Theorems 2.7-2.9, pp. 2021 ), it follows that $G$ may take its nonpositive minimum value under the following three possible cases:

(1) for $t=0$; (2) at a point $(\hat{x}, \hat{t}) \in \Omega \times\left(0, t^{*}\right)$ where $|\nabla u(\hat{x}, \hat{t})|=0$; (3) on the boundary $\partial \Omega \times\left(0, t^{*}\right)$.

We first study the first case. By (2.1), we derive

$$
\begin{aligned}
& \min _{x \in \bar{\Omega}} G(x, 0) \\
& \quad=\min _{x \in \bar{\Omega}}\left\{\nabla \cdot\left(\left|\nabla u_{0}\right|^{p-2} \nabla u_{0}\right)+f\left(x, u_{0},\left|\nabla u_{0}\right|^{2}, 0\right)-\alpha \mathrm{e}^{u_{0}}\right\} \\
& \quad=\min _{x \in \bar{\Omega}}\left\{\mathrm{e}^{u_{0}}\left(\frac{\nabla \cdot\left(\left|\nabla u_{0}\right|^{p-2} \nabla u_{0}\right)+f\left(x, u_{0},\left|\nabla u_{0}\right|^{2}, 0\right)}{\mathrm{e}^{u_{0}}}-\alpha\right)\right\}=0 .
\end{aligned}
$$


Then, we study the second case. With (2.5), we have

$$
\begin{aligned}
G(\hat{x}, \hat{t}) & =\left.\left[\nabla \cdot\left(|\nabla u|^{p-2} \nabla u\right)+f\left(x, u,|\nabla u|^{2}, t\right)-\alpha \mathrm{e}^{u}\right]\right|_{(\hat{x}, \hat{t})} \\
& =\left.\left[|\nabla u|^{p-2} \Delta u+(p-2)|\nabla u|^{p-4} u,{ }_{i} u, j u, i j+f\left(x, u,|\nabla u|^{2}, t\right)-\alpha \mathrm{e}^{u}\right]\right|_{(\hat{x}, \hat{t})} \\
& \geq\left.\left[-|\nabla u|^{p-2}|\Delta u|-(p-2)|\nabla u|^{p-4}|\nabla u||\nabla u||u, i j|+f\left(x, u,|\nabla u|^{2}, t\right)-\alpha \mathrm{e}^{u}\right]\right|_{(\hat{x}, \hat{t})} \\
& =\left.\left[-|\nabla u|^{p-2}|\Delta u|-(p-2)|\nabla u|^{p-2}|u, i j|+\mathrm{e}^{u}\left(\frac{f\left(x, u,|\nabla u|^{2}, t\right)}{\mathrm{e}^{u}}-\alpha\right)\right]\right|_{(\hat{x}, \hat{t})} \\
& =\mathrm{e}^{u(\hat{x}, \hat{t})}\left(\frac{f(\hat{x}, u(\hat{x}, \hat{t}), 0, \hat{t})}{\left.\mathrm{e}^{u(\hat{x}, \hat{t})}-\alpha\right) \geq \mathrm{e}^{u(\hat{x}, \hat{t})}(\beta-\alpha) \geq 0}\right.
\end{aligned}
$$

Finally, we study the third case. Using of the boundary condition of (1.1), we obtain

$$
\frac{\partial G}{\partial n}=b^{\prime \prime} \frac{\partial u}{\partial n} u_{t}+b^{\prime} \frac{\partial u_{t}}{\partial n}-\alpha \mathrm{e}^{u} \frac{\partial u}{\partial n}=b^{\prime}\left(\frac{\partial u}{\partial n}\right)_{t}=0 \quad \text { on } \partial \Omega \times\left(0, t^{*}\right) .
$$

It follows from parabolic maximum principles and (2.22)-(2.24) that the minimum value of $G$ in $\bar{\Omega} \times\left[0, t^{*}\right)$ is zero. In fact, if the minimum value of $G$ in $\bar{\Omega} \times\left[0, t^{*}\right)$ is negative, then this minimum value must be taken on $\partial \Omega \times\left(0, t^{*}\right)$. So there is $(\tilde{x}, \tilde{t}) \in \partial \Omega \times\left(0, t^{*}\right)$ such that $G(\tilde{x}, \tilde{t})=\min _{(x, t) \in \bar{\Omega} \times\left[0, t^{*}\right)} G(x, t)<0$. The parabolic maximum principle means

$$
\left.\frac{\partial G}{\partial n}\right|_{(\tilde{x}, \tilde{t})}<0
$$

which contradicts (2.24). Hence, the minimum value of $G$ in $\bar{\Omega} \times\left[0, t^{*}\right)$ is zero. In other words, we have

$$
G(x, t) \geq 0 \quad \text { in } \bar{\Omega} \times\left[0, t^{*}\right)
$$

from which we get the following differential inequality:

$$
\frac{b^{\prime}(u)}{\alpha \mathrm{e}^{u}} u_{t} \geq 1 \quad \text { in } \bar{\Omega} \times\left[0, t^{*}\right)
$$

At the point $\tilde{x} \in \bar{\Omega}$ where $u_{0}(\tilde{x})=M_{0}$, we integrate (2.25) from 0 to $t$ to get

$$
\frac{1}{\alpha} \int_{0}^{t} \frac{b^{\prime}(u)}{\mathrm{e}^{u}} u_{t} \mathrm{~d} t=\frac{1}{\alpha} \int_{M_{0}}^{u(\tilde{x}, t)} \frac{b^{\prime}(\tau)}{\mathrm{e}^{\tau}} \mathrm{d} \tau \geq t .
$$

It follows from (2.26) that $u$ must blow up at some finite time $t^{*}$. In fact, assuming that the solution $u$ does not blow up, we have, for any $t>0$,

$$
\frac{1}{\alpha} \int_{M_{0}}^{+\infty} \frac{b^{\prime}(\tau)}{\mathrm{e}^{\tau}} \mathrm{d} \tau>\frac{1}{\alpha} \int_{M_{0}}^{u(\tilde{x}, t)} \frac{b^{\prime}(\tau)}{\mathrm{e}^{\tau}} \mathrm{d} \tau \geq t
$$


Letting $t \rightarrow+\infty$ in (2.27), we derive

$$
\frac{1}{\alpha} \int_{M_{0}}^{+\infty} \frac{b^{\prime}(\tau)}{\mathrm{e}^{\tau}} \mathrm{d} \tau=+\infty
$$

which contradicts (2.6). Hence, $u$ must blow up at some finite time $t^{*}$. Furthermore, letting $t \rightarrow t^{*}$ in (2.26), we obtain

$$
t^{*} \leq \frac{1}{\alpha} \int_{M_{0}}^{+\infty} \frac{b^{\prime}(\tau)}{\mathrm{e}^{\tau}} \mathrm{d} \tau
$$

At each fixed point $x \in \bar{\Omega}$, we integrate (2.25) from $t$ to $\check{t}\left(0<t<\check{t}<t^{*}\right)$ and use (2.4) to deduce

$$
H(u(x, t)) \geq H(u(x, t))-H(u(x, \check{t}))=\int_{u(x, t)}^{u(x, \check{t})} \frac{b^{\prime}(\tau)}{\mathrm{e}^{\tau}} \mathrm{d} \tau \geq \alpha(\check{t}-t)
$$

Letting $\check{t} \rightarrow t^{*}$ in (2.28), we have

$$
H(u(x, t)) \geq \alpha\left(t^{*}-t\right)
$$

and

$$
u(x, t) \leq H^{-1}\left(\alpha\left(t^{*}-t\right)\right)
$$

The proof is complete.

In Theorem 2.1, we have the following conclusions when $b(u) \equiv u$ :

Corollary 2.1 Let u be a nonnegative $C^{3}\left(\Omega \times\left(0, t^{*}\right)\right) \cap C^{2}\left(\bar{\Omega} \times\left[0, t^{*}\right)\right)$ solution of the following problem:

$$
\begin{cases}u_{t}=\nabla \cdot\left(|\nabla u|^{p-2} \nabla u\right)+f\left(x, u,|\nabla u|^{2}, t\right) & \text { in } \Omega \times\left(0, t^{*}\right), \\ \frac{\partial u}{\partial n}=0 & \text { on } \partial \Omega \times\left(0, t^{*}\right), \\ u(x, 0)=u_{0}(x) \geq 0 & \text { in } \bar{\Omega},\end{cases}
$$

where $p>2$, the spatial domain $\Omega \subset \mathbb{R}^{N}(N \geq 2)$ is bounded, and the boundary $\partial \Omega$ is smooth. Assume the following two assumptions are true:

(i)

$$
\beta \geq \alpha>0
$$

(ii) $\operatorname{For}(x, s, r, t) \in \Omega \times \overline{\mathbb{R}_{+}} \times \overline{\mathbb{R}_{+}} \times \mathbb{R}_{+}$,

$$
f_{r}(x, s, r, t) \geq 0, \quad f_{t}(x, s, r, t) \geq 0, \quad f_{s}(x, s, r, t)-(p-1) f(x, s, r, t) \geq 0 .
$$


Then $u(x, t)$ must blow up at a finite time $t^{*}$ and

$$
t^{*} \leq \frac{\mathrm{e}^{-M_{0}}}{\alpha}, \quad M_{0}=\max _{x \in \bar{\Omega}} u_{0}(x)
$$

as well as

$$
u(x, t) \leq \ln \frac{1}{\alpha\left(t^{*}-t\right)}
$$

\section{Application}

In the following, we give an example to illustrate the application of Theorem 2.1

Example 3.1 Let $u$ be a nonnegative $C^{3}\left(\Omega \times\left(0, t^{*}\right)\right) \cap C^{2}\left(\bar{\Omega} \times\left[0, t^{*}\right)\right)$ solution of the following problem:

$$
\begin{cases}\left(\mathrm{e}^{\frac{u}{2}}\right)_{t}=\nabla \cdot(|\nabla u| \nabla u)+\left(1+\sum_{i=1}^{3} x_{i}^{2}+|\nabla u|^{2} t\right) \mathrm{e}^{u} & \text { in } \Omega \times\left(0, t^{*}\right), \\ \frac{\partial u}{\partial n}=0 & \text { on } \partial \Omega \times\left(0, t^{*}\right), \\ u(x, 0)=1 & \text { in } \bar{\Omega},\end{cases}
$$

where $\Omega=\left\{x=\left(x_{1}, x_{2}, x_{3}\right) \mid \sum_{i=1}^{3} x_{i}^{2}<1\right\}$. We now have

$$
p=3, \quad b(u)=\mathrm{e}^{\frac{u}{2}}, \quad f\left(x, u,|\nabla u|^{2}, t\right)=\left(1+\sum_{i=1}^{3} x_{i}^{2}+|\nabla u|^{2} t\right) \mathrm{e}^{u}, \quad u_{0}(x)=1 .
$$

It follows from (2.1) and (2.2) that

$$
\alpha=\min _{x \in \bar{\Omega}} \frac{\nabla \cdot\left(\left|\nabla u_{0}\right|^{p-2} \nabla u_{0}\right)+f\left(x, u_{0},\left|\nabla u_{0}\right|^{2}, 0\right)}{\mathrm{e}^{u_{0}}}=\min _{x \in \bar{\Omega}}\left(1+\sum_{i=1}^{3} x_{i}^{2}\right)=1
$$

and

$$
\beta=\inf _{(x, s, t) \in \Omega \times \overline{\mathbb{R}_{+}} \times \mathbb{R}_{+}} \frac{f(x, s, 0, t)}{\mathrm{e}^{s}}=\inf _{(x, s, t) \in \Omega \times \overline{\mathbb{R}_{+}} \times \mathbb{R}_{+}}\left(1+\sum_{i=1}^{3} x_{i}^{2}\right)=1,
$$

from which we know that the assumption (2.5) holds. We also easily check that the assumptions (2.6)-(2.8) hold true. Hence, Theorem 2.1 implies that $u(x, t)$ must blow up at a finite time $t^{*}$ and

$$
t^{*} \leq \frac{1}{\alpha} \int_{M_{0}}^{+\infty} \frac{b^{\prime}(\tau)}{\mathrm{e}^{\tau}} \mathrm{d} \tau=\frac{1}{2} \int_{1}^{+\infty} \mathrm{e}^{-\frac{\tau}{2}} \mathrm{~d} \tau=\mathrm{e}^{-\frac{1}{2}}
$$

as well as

$$
u(x, t) \leq H^{-1}\left(\alpha\left(t^{*}-t\right)\right)=\ln \frac{1}{\left(t^{*}-t\right)^{2}}, \quad(x, t) \in \bar{\Omega} \times\left[0, t^{*}\right) .
$$




\section{Conclusions}

In this paper, we combine parabolic maximum principles with the differential inequality techniques to study the blow-up solution of problem (1.1). The key to our research is constructing two auxiliary functions (2.3) and (2.4). With their help, we obtain sufficient conditions for the existence of the blow-up solution of problem (1.1). In addition, we also give an upper bound on the blow-up time and an upper estimate of the blow-up rate.

\section{Acknowledgements}

The author is sincerely grateful to the editors and anonymous referees for careful reading of the original manuscript and useful comments.

\section{Funding}

This work was supported by the National Natural Science Foundation of China (No 61473180).

\section{Abbreviations}

Not applicable.

Availability of data and materials

Not applicable.

\section{Competing interests}

The author declares that there is no conflict of interests regarding the publication of this paper.

\section{Authors' contributions}

All results are due to JD. All authors read and approved the final manuscript.

\section{Publisher's Note}

Springer Nature remains neutral with regard to jurisdictional claims in published maps and institutional affiliations.

Received: 18 February 2020 Accepted: 21 August 2020 Published online: 01 September 2020

\section{References}

1. Anh, C.T., Ke, T.D.: On quasilinear parabolic equations involving weighted p-Laplacian operators. Nonlinear Differ. Equ. Appl. 17, 195-212 (2010)

2. Ding, J.T.: Global existence and blow-up results for $p$-Laplacian parabolic problems under nonlinear boundary conditions. J. Inequal. Appl. 2018, 67 (2018)

3. Ding, J.T.: Blow-up phenomena for p-Laplacian parabolic problems with Neumann boundary conditions. Bound. Value Probl. 2017, 149 (2017)

4. Ding, J.T., Shen, X.H.: Blow-up in p-Laplacian heat equations with nonlinear boundary conditions. Z. Angew. Math. Phys. 67, 125 (2016)

5. Le, C.N., Le, X.T.: Global solution and blow-up for a class of p-Laplacian evolution equations with logarithmic nonlinearity. Acta Appl. Math. 151, 149-169 (2017)

6. Li, F.S., Li, J.L.: Global existence and blow-up phenomena for p-Laplacian heat equation with inhomogeneous Neumann boundary conditions. Bound. Value Probl. 2014, 219 (2014)

7. Lian, S.Z., Yuan, H.J., Cao, C.L., Gao, W.J., Xu, X.J.: On the Cauchy problem for the evolution p-Laplacian equations with gradient term and source. J. Differ. Equ. 235, 544-585 (2007)

8. Liu, W.J.: A note on blow-up for fast diffusive p-Laplacian with sources. Math. Methods Appl. Sci. 32, $241-245$ (2009)

9. Lu, H.Q., Zhang, Z.G.: Blow-up time estimate for a parabolic p-Laplacian equation with nonlinear gradient terms. Z. Angew. Math. Phys. 70, 3 (2019)

10. Yin, J.X., Jin, C.H.: Critical extinction and blow-up exponents for fast diffusive p-Laplacian with sources. Math. Methods Appl. Sci. 30, 1147-1167 (2007)

11. Andreu, F., Mazon, J.M., Simondon, F., Toledo, J.: Global existence for a degenerate nonlinear diffusion problem with nonlinear gradient term and source. Math. Ann. 314, 703-728 (1999)

12. Ding, J.T., Guo, B.Z.: Global existence and blow-up solutions for quasilinear reaction-diffusion equations with a gradient term. Appl. Math. Lett. 24, 936-942 (2011)

13. Ding, J.T.: Blow-up solutions for a class of nonlinear parabolic equations with Dirichlet boundary conditions. Nonlinear Anal. 52, 1645-1654 (2003)

14. Guo, J.S., Hu, B.: Blowup rate estimates for the heat equation with a nonlinear gradient source term. Discrete Contin. Dyn. Syst. 20, 927-937 (2008)

15. Payne, L.E., Philippin, G.A., Vernier-Piro, S.: Blow up, decay bounds and continuous dependence inequalities for a class of quasilinear parabolic problems. Math. Methods Appl. Sci. 29, 281-295 (2006)

16. Quittner, P.: Blow-up for semilinear parabolic equations with a gradient term. Math. Methods Appl. Sci. 14, 413-417 (1991)

17. Snoussi, S., Tayachi, S.: Large time behavior of solutions for parabolic equations with nonlinear gradient terms Hokkaido Math. J. 36, 311-344 (2007)

18. Souplet, P.: Gradient blow-up for multidimensional nonlinear parabolic equations with general boundary conditions. Differ. Integral Equ. 15, 237-256 (2002) 
19. Souple, P., Tayachi, S.: Blowup rates for nonlinear heat equations with gradient terms and for parabolic inequalities. Colloq. Math. 88, 135-154 (2001)

20. Tayachi, S., Zaag, H.: Existence of a stable blow-up profile for the nonlinear heat equation with a critical power nonlinear gradient term. Trans. Am. Math. Soc. 371, 5899-5972 (2019)

21. Zhang, L.L., Tian, H.M.: Blow-up phenomena for a class of nonlinear reaction-diffusion equations under nonlinear boundary conditions. Appl. Anal. 98, 2868-2883 (2019)

22. Zhang, L.L., Wang, H., Wang, X.Q.: Global and blow-up analysis for a class of nonlinear reaction diffusion model with Dirichlet boundary conditions. Math. Methods Appl. Sci. 41, 7789-7803 (2018)

23. Zhao, J.N.: Existence and nonexistence of solutions for $u_{t}=\operatorname{div}\left(|\nabla u|^{p-2} \nabla u\right)+f(\nabla u, u, x, t)$. J. Math. Anal. Appl. 172, 130-146 (1993)

24. Sperb, R.P.: Maximum Principles and Their Applications. Academic Press, New York (1981)

Submit your manuscript to a SpringerOpen ${ }^{\circ}$ journal and benefit from:

- Convenient online submission

- Rigorous peer review

Open access: articles freely available online

- High visibility within the field

- Retaining the copyright to your article

Submit your next manuscript at $\boldsymbol{\nabla}$ springeropen.com 